\title{
DYNAMIC ATTENTION AS A PREDICTOR OF DRIVING PERFORMANCE IN CLINICAL POPULATIONS: PRELIMINARY RESULTS
}

\author{
Alex Bowers ${ }^{1}$, Julius Anastasio'1, Piers Howe ${ }^{2}$, Margaret O’Connor ${ }^{3}$, \\ Ann Hollis ${ }^{3}$, Lissa Kapust ${ }^{3}$, Matt Bronstad ${ }^{1}$, \& Todd Horowitz ${ }^{4}$ \\ ${ }^{1}$ The Schepens Eye Research Institute, Harvard Medical School, Boston, Massachusetts, USA \\ ${ }^{2}$ Psychological Sciences, University of Melbourne, Melbourne, Victoria, Australia \\ ${ }^{3}$ Department of Neurology, Beth Israel Deaconess Medical Center \\ ${ }^{4}$ Brigham and Women's Hospital, Harvard Medical School \\ Boston, Massachusetts, USA \\ Email: alex.bowers@schepens.harvard.edu
}

\begin{abstract}
Summary: Existing tests (e.g., useful field of view; UFOV) that are commonly used to evaluate visual attention when predicting at-risk drivers do not have a dynamic component. In this project, we developed a brief computerized test of dynamic visual attention (multiple object tracking; MOT). Estimates of threshold tracking speed from the brief MOT test showed good agreement with those determined by a full psychometric function $(\mathrm{n}=41, \mathrm{r}=0.876, \mathrm{p}<0.001)$. The brief MOT test was then implemented in a clinical driving assessment program; participants with poorer MOT scores had higher error scores on the road test $(n=$ $15, \mathrm{r}=-0.670, \mathrm{p}=0.006)$.
\end{abstract}

\section{BACKGROUND AND OBJECTIVES}

Driving is a complex task that involves visual processing of a constantly changing, dynamic environment. Age-related impairments in cognition and/or visual function may affect the ability to deploy attention when driving, with adverse consequences for the driver and other road users. The primary emphasis in driver screening is on testing visual acuity, and to some extent measuring visual fields, while the cognitive elements of driving are often ignored. Unfortunately, visual acuity turns out to be a poor predictor of driving performance, and many studies have found little association between crash rates and visual field deficits (for a review, see Owsley \& McGwin, 2010).

Visual attention is an important component of many driving tasks. Over the last decade or so, researchers have established a connection between measures of visual attention and driving (Baldock, Mathias, McLean, \& Berndt, 2007; Ball, Beard, Roenker, Miller, \& Griggs, 1988). Particularly successful has been the Useful Field of View test (UFOV, Ball et al., 1988; Hoffman, McDowd, Atchley, \& Dubinsky, 2005). However, while there are significant associations between UFOV and driving performance (Clay et al., 2005), ROC analysis (Bedard, Weaver, Darzins, \& Porter, 2008) suggests that as a screening test it would produce unacceptable numbers of false positives (unsafe drivers categorized as safe) and/or false negatives (safe drivers categorized as unsafe).

We suggest that there are two aspects of attentional function not tested by the UFOV that may be critical to improving prediction of driving performance and accident propensity. First, the UFOV only tests selective and divided attention. It does not require sustained attention, since stimuli are 
presented briefly, for durations well under a second. Second, and perhaps more importantly, the UFOV stimuli are static. Driving, however, must be carried out in a dynamic environment in which both the observer and the objects in the environment are in motion. Therefore, it seems plausible that a task requiring sustained attention to moving stimuli would provide advantages over the UFOV and the family of similar attention tests that use brief exposures and static stimuli (e.g., DriverScan, Hoffman et al., 2005).

The multiple object tracking (MOT, Pylyshyn \& Storm, 1988) task meets these criteria (for reviews, see Cavanagh \& Alvarez, 2005; Scholl, 2009). In a typical MOT experiment, observers are presented with an array of identical objects, such as disks. Some of these objects are designated as targets. The objects then move independently for several seconds (or minutes, see Wolfe, Place, \& Horowitz, 2007). The observer must indicate which items at the end of the trial correspond to the targets identified at the beginning. MOT thus captures all three aspects of visual attention. Observers must select targets over distractors, divide attention across targets, and sustain attention for the tracking duration. Furthermore, since the objects are continually in motion, MOT captures the dynamic aspects of driving. In addition to this high face validity, MOT has been shown to be sensitive to factors known to affect driving, including age (Sekuler, McLaughlin, \& Yotsumoto, 2008; Trick, Perl, \& Sethi, 2005), sleepiness (Horowitz, Wolfe, Cohen, Czeisler, \& Klerman, 2008), telephone conversation, and verbal generation tasks in general (Kunar, Carter, Cohen, \& Horowitz, 2008). However, the relationship between driving and MOT has never been established.

We hypothesize that MOT captures the key attentional skills required for driving, and thus will be a useful predictor of driving performance. To test this hypothesis, we developed a brief MOT test (section 1) and then implemented it in a clinical driving assessment program (section 2).

\section{SECTION 1: DEVELOPMENT AND VALIDATION STUDY}

We had two design goals. First, while laboratory-based MOT sessions typically take 60 minutes to complete (e.g, Horowitz et al., 2007), a practical screening test to be used with older people should be fairly brief. Second, we wanted a task which would produce meaningful results across a wide range of tracking ability. Therefore, instead of using accuracy at a fixed speed as a dependent variable, as is typical, we measured the speed at which observers could track at a given accuracy level using an adaptive method. For validation purposes, threshold estimates from this brief task were compared to estimates obtained by measuring the full psychometric function (MOT-PF). Studies were approved by IRB committees at Schepens Eye Research Institute and Beth Israel Deaconess Medical Center. Data analyses were performed with SPSS, version 11.5 (SPSS, 2002).

Apparatus. Visual stimuli were presented on 20-in. color LCD touchscreen monitors (NEC MultiSync LCD2090UXi) controlled by computers with AMD Phenom II X3 710 Processors (Microsoft OS Windows XP Professional SP3 ) running Matlab version 7.5.0.342 using the Psychophysics Toolbox (Brainard, 1997; Pelli, 1997). Monitor spatial resolution was set at $1600 x 1200$ pixels. Monitor refresh rates were set to $60 \mathrm{~Hz}$ (16.66msec per frame). 
Task. Stimuli were six high contrast black disks, subtending $2^{\circ}$ visual angle at the $42 \mathrm{~cm}$ viewing distance, presented against a lighter gray background. At the start of each trial, three target disks were highlighted in green for $2 \mathrm{~s}$. The observer had to track the target disks as they moved for 5$8 \mathrm{~s}$. Each disk was assigned an initial random direction. Disks changed direction only when they met the boundaries of the display $\left(20^{\circ} \times 20^{\circ}\right)$ or a buffer zone one disk width in radius around any other disk. Thus, disks never occluded one another. At the end of the trial, all disks stopped moving and the observer used the touchscreen to indicate which disks were targets. Once three disks had been selected, target and distracter disks were revealed, with the observer's selections highlighted in red.

Measuring speed thresholds. We first ran the brief version of the MOT task (brief MOT). In this version, speeds were adjusted on each trial using a simple one-up, one-down staircase. The initial speed was set at $12 \%$ s. Speed was increased by $40 \%$ following correct trials, and decreased by $60 \%$ following incorrect trials, ensuring that a wide range of speeds was sampled. There were 50 trials; the task took approximately 15-20 min. In the MOT-PF, we measured accuracy at 7 different speeds, logarithmically spaced around the $60 \%$ threshold estimated in the brief MOT, chosen to span the entire psychometric function. Speeds were randomly intermixed from trial to trial, with 10 trials for each speed. In both versions, all targets had to be identified correctly in order for the trial to be counted as correct. We computed the $60 \%$ threshold in both cases using the QUEST algorithm (King-Smith, Grigsby, Vingrys, Benes, \& Supowit, 1994; Watson \& Pelli, 1983).

Participants and procedure. 41 healthy subjects (age: mean 59 (SD 22) years, range 22 to 85) completed first the brief MOT and then the MOT-PF.

\section{Results}

Threshold speeds determined by the brief MOT and MOT-PF tasks were highly correlated (Pearson's $r=0.876, \mathrm{p}<0.001$; Figure 1a) with an average difference in the mean values of only $0.2 \%$ (95\% CI limits -0.6 and 0.9 , respectively; $\mathrm{t}_{(40)}=0.41, \mathrm{p}=0.684$; Figure $\left.1 \mathrm{~b}\right)$.

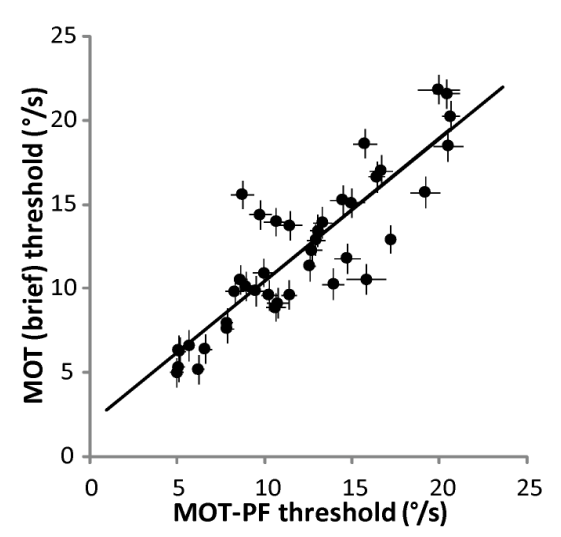

Figure 1a. Threshold tracking speeds determined from the brief MOT task plotted against thresholds determined from the full psychometric function (MOT-PF) Error bars represent standard deviations from the QUEST algorithm for each threshold speed

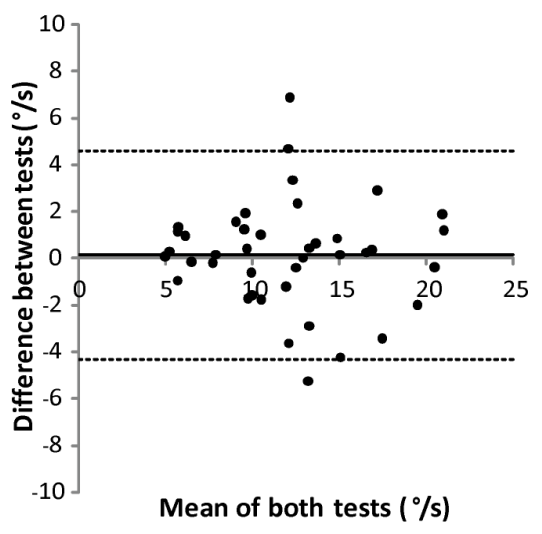

Figure 1b. Differences in threshold speeds determined by the two MOT tests

The solid line is the mean of the differences, the dashed lines are the $95 \%$ limits of agreement (Bland \& Altman, 1986) 


\section{SECTION 2: CLINICAL IMPLEMENTATION STUDY}

\section{Participants}

Participants were selected from all patients undergoing comprehensive evaluations through the DriveWise Clinical Program at BIDMC (O'Connor, Kapust, \& Hollis, 2008). Patients were included in the study if they were aged 50 years or older, possessed a valid driver's license, had visual acuity of at least 20/40, met the visual field requirements for licensure in MA (at least $120^{\circ}$ horizontal diameter), possessed no physical impairments, and completed the DriveWise assessment. We report data from the first 17 patients (10 male, 7 female) who met the inclusion criteria (age: mean $=80$ (SD 9.9) years, range $=58-94$, years). Six of the participants had mild cognitive impairment, as defined by their clinical diagnoses and MMSE scores (Crum, Anthony, Bassett, \& Folstein, 1993).

\section{Methods}

Clinical evaluation. An occupational therapist (OT) administered tests of physical function, cognitive skills, and vision. The MMSE, UFOV, and brief MOT tests were included as part of the clinical evaluation. Participants first practiced the MOT task at a fixed threshold speed of $2 \% \mathrm{~s}$ until they were familiar with the task. Participants then underwent 10 practice trials of the brief MOT task before completing the 50 experimental trials. The MOT test was administered to all participants, but one participant did not do the UFOV test because of insufficient time.

On-road evaluation. After the clinical evaluation, each participant underwent a 45 minute (7.5 miles) standardized road test modeled after the Washington University Road Test (Hunt et al, 1997). The route was conducted on urban, local and highway roads. All driving tests were completed between 1 and 3pm. Tests were conducted by an occupational therapist (OT) and a certified driving rehabilitation specialist (DRS) in a dual-control car. The DRS provided directions and monitored overall safety, while the OT sat in the back seat and recorded behavioral performance for a range of maneuvers (right turn, left turn, merge, change lane) at pre-determined locations along the route. For each maneuver, a standardized set of skill items (including speed, lane position, gap selection, observation etc.) were scored on a binary scale: "pass" and "error." If a driving error or a verbal and/or physical intervention occurred within a maneuver item, the participant was classified as having made an error on that item. For each participant, the total number of items with an error was computed and expressed as a proportion of the total number of items scored. Due to safety concerns, one participant's road test was terminated by the DRS during the practice period; therefore detailed scoring of errors for specific maneuvers was not performed. The DRS had no knowledge of participants' scores on the test battery (MMSE, UFOV, MOT) and the OT had no knowledge of participants' MOT scores.

Analyses. We used Pearson's correlations to quantify the relationship between MOT scores, UFOV scores and proportion of total driving errors for scored maneuvers. Only the divided attention score from the UFOV test was included as it is the subtest reported to have the strongest association with driving performance (Ball et al., 2006). Note that a high score (faster speed) on the MOT test represents good performance whereas a high score (long presentation time) on the UFOV divided attention task represents poor performance. 


\section{Preliminary Results}

Performance on the brief MOT test. There was a wide range of performance on the brief MOT test with threshold speeds ranging from $0 \%$ s to $18 \%$. Two participants with mild cognitive impairment had speeds of $0 \% \mathrm{~s}$. The first gave incorrect responses on all trials and the task was discontinued after the threshold reached $1.0^{-04} \%$ s. The second gave correct responses on some trials and completed the brief MOT task, but received a threshold of $1.2^{-09} \% \mathrm{~s}$. Nevertheless, their data are included as they were unable to perform the dynamic tracking task due to cognitive attentional impairments rather than visual or motor impairments. The participant for which the road test was terminated during the practice period had a speed threshold of $5.4 \% \mathrm{~s}$ on the brief MOT test.

Relationship between brief MOT score, UFOV score and driving performance. There were moderate correlations between the proportion of total driving errors and scores on both the brief MOT task $(\mathrm{n}=15, \mathrm{r}=-0.670, \mathrm{p}=0.006$; Figure $2 \mathrm{a})$ and the UFOV divided attention task $(\mathrm{n}=$ $15, r=0.656, p=0.008$; Figure $2 b$ ). Scores on the MOT and UFOV tasks were also significantly correlated $(\mathrm{n}=15 \mathrm{r}=-0.596, \mathrm{p}=0.015$; Figure $2 \mathrm{c})$.

A stepwise multiple regression analysis was performed with proportion of driving errors as the dependent variable, and MOT score, UFOV divided attention score, age and cognitive status (impaired/non-impaired) as independent variables. MOT score was selected as the only significant predictor, accounting for $41 \%$ of the variance in the proportion of driving errors $(\mathrm{p}=$ 0.006). Forcing UFOV into the regression equation only accounted for another $7 \%$ of the variance.
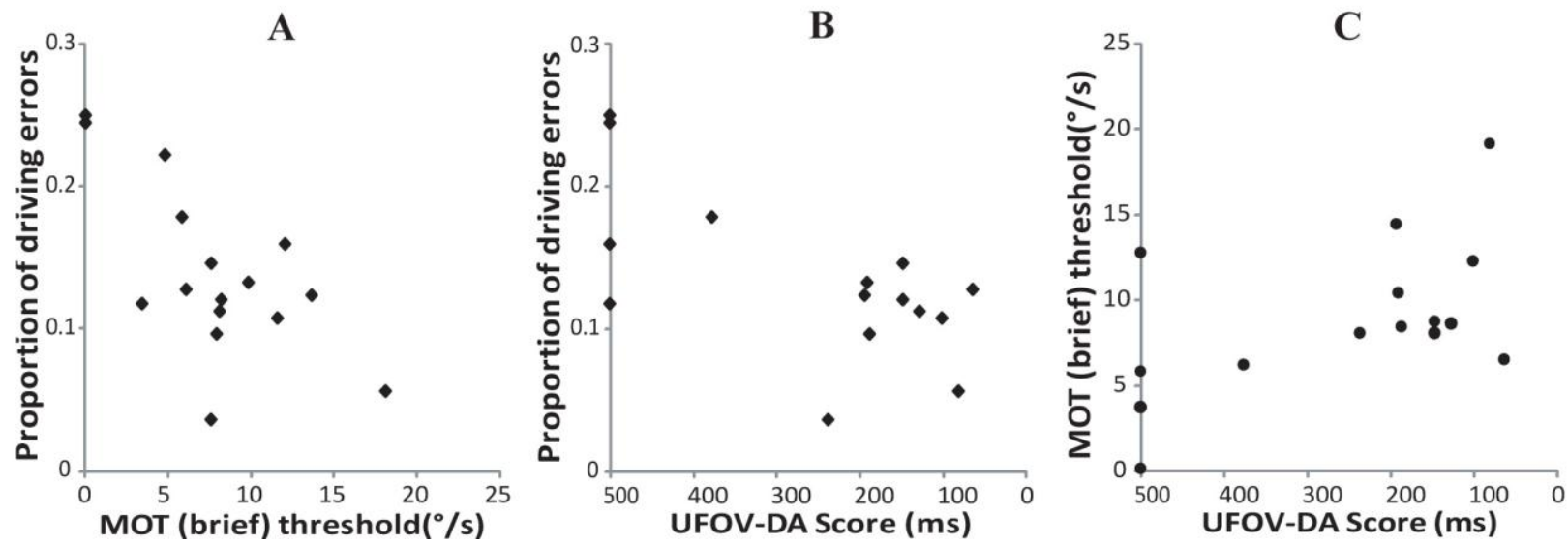

Figure 2. Relationship between MOT, UFOV divided attention and driving performance: (a) MOT and proportion of driving errors, (b) UFOV and proportion of driving errors, (c) UFOV and MOT (Both MOT data and UFOV data are plotted from poor to good (0 to $25 \%$ s and 500 to $17 \mathrm{~ms}$, respectively)

\section{DISCUSSION}

We successfully developed a brief, adaptive test of dynamic attention and applied it in a clinical driving assessment program. The adaptive procedure provides a robust estimate of the speed threshold with fewer trials than would be needed for measuring the full psychometric function. Preliminary results demonstrate a wide range of performance within a clinical population representative of older drivers likely to be evaluated for driving safety. Two participants had 
tracking speeds of $0 \%$, suggesting they were unable to track at all. This might be seen as a weakness of the test. However, in the context of developing a screening test to identify at-risk drivers, an inability to track suggests significant impairments in memory and attention. In support of this argument, the two participants with thresholds of $0 \% \mathrm{~s}$ had the highest proportion of driving errors and were both rated as unsafe drivers.

Our preliminary results demonstrate a moderate correlation between MOT speed thresholds and overall driving performance; participants with poorer MOT scores had higher error rates on the road test. The strength of the correlation was similar to that between UFOV divided attention and driving performance. MOT and UFOV were also moderately correlated, suggesting that the two tests evaluate common aspects of attentional function (presumably divided attention). However, the data also suggest that MOT may capture aspects of attention not evaluated by the UFOV test, e.g., participants with UFOV performance at floor (threshold of 500ms) had MOT scores ranging from very poor $(0 \%$ s) to relatively good $(12 \%$; Figure $2 \mathrm{c})$.

Although these are early results and from a small sample, they are promising and suggest that our brief MOT test might be a useful predictor of driving performance in older people. We are continuing to collect data to increase our sample size and address the question of whether the brief MOT test is more predictive of driving test outcome (pass/fail on a road test) than other commonly-used tests of cognition and attention.

\section{ACKNOWLEDGEMENTS}

Funded in part by NIH grant EY018680 (AB) and a pilot grant from Harvard Catalyst, The Harvard Clinical and Translational Science Center (NIH Grant \#1 UL1 RR 025758-02).

\section{REFERENCES}

Baldock, M. R. J., Mathias, J., McLean, J., \& Berndt, A. (2007). Visual attention as a predictor of on-road driving performance of older drivers. Australian Journal of Psychology, 59(3), 159-168.

Ball, K. K., Beard, B. L., Roenker, D. L., Miller, R. L., \& Griggs, D. S. (1988). Age and visual search: expanding the useful field of view. Journal of the Optical Society of America $(A)$, 5(12), 2210-2219.

Ball, K. K., Roenker, D. L., Wadley, V. G., Edwards, J. D., Roth, D. L., McGwin, G., et al. (2006). Can high-risk older drivers be identified through performance-based measures in a department of motor vehicles setting? Journal of the American Geriatrics Society, 54(1), 77-84.

Bedard, M., Weaver, B., Darzins, P., \& Porter, M. M. (2008). Predicting Driving Performance in Older Adults: We Are Not There Yet! Traffic Injury Prevention, 9(4), 336-341.

Bland, J. M., \& Altman, D. G. (1986). Statistical methods for assessing agreement between two methods of clinical measurement. Lancet, 1(8476), 307-310.

Brainard, D. H. (1997). The Psychophysics Toolbox. Spatial Vision, 10, 443-446.

Cavanagh, P., \& Alvarez, G. A. (2005). Tracking multiple targets with multifocal attention. Trends in Cognitive Sciences, 9(7), 349-354. 
Clay, O. J., Wadley, V. G., Edwards, J. D., Roth, D. L., Roenker, D. L., \& Ball, K. K. (2005). Cumulative meta-analysis of the relationship between useful field of view and driving performance in older adults: Current and future implications. Optometry and Vision Science, 82(8), 724-731.

Crum, R. M., Anthony, J. C., Bassett, S. S., \& Folstein, M. F. (1993). Population-Based Norms for the Mini-Mental-State-Examination by Age and Educational-Level. Jama-Journal of the American Medical Association, 269(18), 2386-2391.

Hoffman, L., McDowd, J. M., Atchley, P., \& Dubinsky, R. (2005). The role of visual attention in predicting driving impairment in older adults. Psychology and Aging, 20(4), 610-622.

Horowitz, T. S., Klieger, S. B., Fencsik, D. E., Yang, K. K., Alvarez, G. A., \& Wolfe, J. M. (2007). Tracking unique objects. Perception \& psychophysics, 69(2), 172-184.

Horowitz, T. S., Wolfe, J., Cohen, D., Czeisler, C., \& Klerman, E. (2008). Quantifying the effects of sleepiness on sustained visual attention (abstract). Journal of Vision, 8(6), 233.

King-Smith, P. E., Grigsby, S. S., Vingrys, A. J., Benes, S. C., \& Supowit, A. (1994). Efficient and unbiased modifications of the QUEST threshold method: Theory, simulations, experimental evaluation and practical implementation. Vision Research, 34(7), 885-912.

Kunar, M. A., Carter, R., Cohen, M., \& Horowitz, T. S. (2008). Telephone conversation impairs sustained visual attention via a central bottleneck. Psychonomic Bulletin \& Review, 15(6), 1135-1140.

O'Connor, M. L., Kapust, L., \& Hollis, A. (2008). DriveWise: An interdisciplinary hospitalbased driving assessment program. . Gerontology \& Geriatrics Education, 29(4), 351-362.

Owsley, C., \& McGwin, G. (2010). Vision and driving. Vision Research, 50, 2348-2361.

Pelli, D. G. (1997). The VideoToolbox software for visual psychophysics: transforming numbers into movies. Spatial Vision, 10(4), 437-442.

Pylyshyn, Z. W., \& Storm, R. W. (1988). Tracking multiple independent targets: Evidence for a parallel tracking mechanism. Spatial Vision, 3, 179-197.

Scholl, B. J. (2009). What have we learned about attention from multiple object tracking (and vice versa)? In D. Dedrick \& L. Trick (Eds.), Computation, Cognition, and Pylyshyn (pp. 49 - 78). Cambridge, MA: MIT Press.

Sekuler, R., McLaughlin, C., \& Yotsumoto, Y. (2008). Age-related changes in attentional tracking of multiple moving objects. Perception, 37(6), 867-876.

Trick, L. M., Perl, T., \& Sethi, N. (2005). Age-related differences in multiple-object tracking. Journals of Gerontology Series B-Psychological Sciences and Social Sciences, 60(2), 102105.

Watson, A. B., \& Pelli, D. G. (1983). QUEST: A Bayesian adaptive psychometric method. Perception and Psychophysics, 33(2), 113-120.

Wolfe, J. M., Place, S. S., \& Horowitz, T. S. (2007). Multiple object juggling: Changing what is tracked during extended multiple object tracking. Psychonomic Bulletin \& Review, 14(2), 344-349. 